\title{
KOMPLEXITÄTEN UND REDUKTIONEN. Zu einigen Prämissen der Popmusikanalyse*
}

\section{Simon Obert}

Die Situation, in der sich die Diskussion der Analyse populärer Musik befindet, ist vordergründig so paradox wie grundsätzlich notwendig: Zweifellos ist Analyse ein virulentes Thema. Blättert man beispielsweise das Register im 2009 von Derek B. Scott herausgegebenen Ashgate Research Companion to Popular Musicology durch, sieht man, dass von allen Stichwörtern unter »analysis« die meisten Seitenverweise eingetragen sind - und das, obwohl von den 26 Essays des immerhin knapp 500 Seiten umfassenden Bandes lediglich einer explizit der Analyse gewidmet ist. Gleichzeitig scheint mit der Virulenz der Thematik auch schon das Ende des Konsens' erreicht zu sein. Sichtet und vergleicht man die zur Analyse populärer Musik erschienene Literatur, erfährt man vor allem eines: dass in den einzelnen Punkten, die die Analyse differenzieren und spezifizierenden - sie damit notwendigerweise vom Rang einer bloß allgemeinen Forderung auf eine praktische Ebene sherunterkonkretisieren< - , Dissens besteht. Was sind überhaupt die konkret zu analysierenden Gegenstände - Klänge, Strukturen, Stücke, Werke, soziokulturelle Akte, Diskurse? Was sind, hinsichtlich des Gegenstands, angenommen, man habe inn gefunden bzw. festgelegt, die angemessenen Methoden der Analyse? Was sind die für die jeweilige Analysemethode sowohl notwendigen als auch passenden Theorien, auf denen man aufbauen kann und mittels derer man sich in einem Forschungsdiskurs verortet? Was sind hinsichtlich eines Analyse-Gegenstands die angemessenen Termini, Idiome, Textsorten, grafischen und anderen Mittel, um die Ergebnisse darzustellen? Wie grenzt man eine jeweilige analytische Fragestellung ein, um nicht ins Uferlose zu geraten und somit eine wünschenswerte Klarheit zu erzielen? Wie weitet man sie aus, um sich notwendigerweise verständlich zu machen - konkret: wie trägt man mit Analyse(n) zum Forschungsdiskurs bei? An wen richtet man sich damit? Wer liest überhaupt Analysen und warum? -

* Für anregende Gespräche danke ich Sarah Chaker (Wien), Maria Hanáček (Berlin) und David Nicholls (Southampton). 
Offene Fragen sind freilich das Brot einer Wissenschaft. Sie sind konstitutiv, weil es zum reflektiven Selbstverständnis der Geistes- und Sozialwissenschaften gehört, nichts a priori anzunehmen. Und diese kritische Grundhaltung bezieht sich sowohl auf die Forschungsobjekte der Wissenschaften als auch auf ihr eigenes Tun.

Aus den Debatten zur Analyse populärer Musik seien im Folgenden vier Themenfelder aufgegriffen: zum ersten die Frage nach dem Gegenstand der Analyse, dahingehend spezifiziert, in welcher Relation »Text« und »Kontext « zueinander stehen; zum zweiten das immer wieder geforderte Postulat der Angemessenheit von Analyse-Methode und analysierter Musik; zum dritten das Problem der Notation bzw. grafischen Repräsentation; und schließlich der Anspruch, was Analyse leisten könne. Die letzten drei der genannten Themen seien aus einer bestimmten Perspektive betrachtet: Es ist auffällig, dass innerhalb der skizzierten offenen Situation ungeklärter Fragen unter Popmusikforschern eine gewisse Einigkeit darin besteht, wie Popanalyse nicht gemacht werden solle. Als argumentative Strategie dienen in methodologischen Texten denn auch häufig die Historische Musikwissenschaft und Musiktheorie als Negativfolie, um über die Abgrenzung von diesen zu verdeutlichen, welche Fehler zu vermeiden sind bzw. welche Irrtümer und falschen Schlussfolgerungen Popmusikanalyse sich einhandelte, würde sie deren Methoden und damit theoretische und ästhetische Implikationen unreflektiert übernehmen. Diese Abgrenzungsstrategie ist für das Selbstverständnis der Popularmusikforschung im Rahmen einer fortdauernden Selbstkonstitution als nach wie vor junge Disziplin einerseits so sinnvoll, wie es andererseits das Abgegrenzte, die meistens nur herbeizitiert »herkömmliche "Musikwissenschaft, im Prinzip auf das "Beethoven paradigm« (Goehr 1992: 205) verkürzt. Diese abgrenzende Kritik sei daher hinsichtlich der drei genannten Themen auf ihre Prämissen befragt. Sollte es sich zeigen, dass bereits die Prämissen nicht zutreffen, dann ist zwar die Kritik nicht unbedingt wertlos, aber sie hat wahrscheinlich andere Ursachen und müsste demnach auf etwas anderes zielen, als sie vorgibt. Denn manche der in den Debatten angesprochenen Problem- und Fragestellungen, mit denen Popmusikanalyse umzugehen hat, dürften weniger aus einer sogenannten »inadäquaten« Übertragung von sogenannten »herkömmlichen« Methoden, Terminologien und Darstellungsweisen resultieren. Vielmehr sind dies Probleme und Fragen, die die musikalische Analyse allgemein betreffen, unabhängig von einer wissenschaftsdisziplinären Ausrichtung nach popmusikalischen, historischen, systematischen, ethnologischen oder theoretischen Schwerpunkten. 


\section{Zum Gegenstand, oder: Is There a Text/Context in This Context/Text?}

Die Frage nach dem Gegenstand musikalischer Analyse ist eine der dringlichsten, angesichts des vielschichtigen und vielgestaltigen Handlungs- und Gegenstandskomplexes, der Musik konstituiert. Die nahe liegende Entscheidung, zwischen klingendem Text, der der musikalischen Analyse zugänglich ist, und nicht klingendem Kontext, der aber konstitutiv zu einer Musik gehört, weil sie innerhalb dieses Kontextes entstanden sei sowie rezipiert wurde und werde, zu unterscheiden, ist eher einer Pragmatik geschuldet, als dass sie objektive Definition wäre. Denn zum einen ist das, was klingt, ein Wahrnehmungsgegenstand und demnach vom jeweiligen Hörer abhängig. Der Hörer konstituiert seinen Hörgegenstand. Dies ist keine Frage der Metaphorik - es ist eine Frage, die einen Gegenstand, je nach Art der Wahrnehmung, tatsächlich ändern kann. Beispielsweise analysiert Dörte HartwichWiechell in ihrem Buch Pop-Musik. Analysen und Interpretationen neben anderen den Song "Street Fighting Man« (1968) der Rolling Stones. In ihrer Transkription des Outros, das nach dem dritten Chorus beginnt (ab 2:30), notiert sie einen durchgehenden schwarzen Balken und schreibt dazu: »elektronisch erzeugter Liegeton« (Hartwich-Wiechell 1974: 371). Mittlerweile weiß man, dass das, was dort erklingt, von einer Shehani, einem indischen Doppelrohrblattinstrument, stammt. Ein Hörer, dem dieses Wissen zur Verfügung steht (sei es über andere oder aus eigener Erfahrung erworben), hört den Song dementsprechend anders als jemand, der dies nicht weiß oder den Klang nicht identifizieren kann. ${ }^{1}$ Und damit ist der Gegenstand auch ein anderer. Denn die wahrnehmungsbedingte Seinsweise eines Gegenstands lässt sich von seinen »Erscheinungen nicht trennen«, weil »zu den Erscheinungen eines Objekts lediglich das [zählt], was wir im Medium unserer Sinne an ihm unterscheiden können« (Seel 2003: 70f.). Würde man daraus den relativierenden Schluss ziehen, dass alle Wahrnehmung nur vorläufig und deswegen in letzter Konsequenz unhaltbar sei, hieße das, sich auf einen irgendwo hinter der Wahrnehmung befindlichen Gegenstand zurückzuziehen und damit einem Objektivismus das Wort reden, demzufolge musikalische Stücke unveränderliche Entitäten darstellten. Objekte menschlicher Wahrnehmung bestehen aber nur zu jeweils subjektiven und sozialen, d.h. intersubjektiv verhandelten Bedingungen. Die unterschiedliche Wahrnehmungs-

1 Das Beispiel soll lediglich zur Verdeutlichung der Konstitution des Wahrnehmungsgegenstands durch den Hörer dienen; eine negative Darstellung von Hartwich-Wiechell liegt mir fern. 
weise bzw. Gegenstandskonstitution, ob in »Street Fighting Man« ein elektronischer oder ein Shehani-Klang zu hören ist, hat weitreichende Konsequenzen: Ändert sich der wahrgenommene Klang, ändert sich auch der Gegenstand.

Ist demnach gar nicht so klar, was mit einem Text gemeint ist, ist es auf der anderen Seite, der des Kontextes, keineswegs klarer. Was für den einen ein Text ist, mag für den anderen ein Kontext sein und umgekehrt, je nach Erkenntnisinteresse (oder disziplinärem Hintergrund). Für einen Musikwissenschaftler, der die Verwendung fernöstlicher Instrumente in populärer Musik untersucht, mag der Song "Street Fighting Man« ein Text sein, für einen Kulturwissenschaftler, der die '68er-Bewegung untersucht, kann er ein Kontext sein. Daher ist, kurz gesagt, als Kontext je das zu begreifen, was unter erkenntnistheoretischen (oder allgemeiner: interessegeleiteten) Prämissen je kontextualisiert wird: Kontexte sind nicht per se vorhanden - sie umgeben nicht einen Text wie das Wasser den Fisch -, sondern sind als solche überhaupt erst zu erkennen. So gesehen ist das Erkennen und Zuschreiben von Kontexten prinzipiell unabschließbar. Gewiss gibt es nahe liegende Kontexte, sofern sie eine mehrheitlich verfügbare Erfahrung oder ein akzeptiertes Wissen darstellen wie z.B. Stil oder Genre. Dennoch ist die Zuordnung eines Musikstücks zu einem Genre eine vorzunehmende Leistung, egal ob sie unbewusst oder bewusst geschieht. So wie das, was man hört (»Text«) vom Hörer abhängig ist, so ist das, was als jeweiliger Kontext erkannt und zugeordnet wird, vom Rezipienten abhängig. Daraus ist zu folgern, dass Text und Kontext nicht zu trennen sind, weil sie in einem gegenseitigen Konstituierungsverhältnis stehen. Ein Musikstück wird ebenso durch seinen Kontext bestimmt, wie es diesen (mit-)bestimmt: Ändert sich der Kontext durch unterschiedliche Kontextualisierungen, ändert sich damit einhergehend die Musik; ändert sich die Musik durch unterschiedliche Wahrnehmungs- und Verstehensweisen, ändert sich auch der Kontext.

Dies sei anhand des Songs "Street Fighting Man« kurz skizziert: Wenn man im Outro einen elektronisch erzeugten Klang hört, würde das Aussagen implizieren über den technologischen Stand von Tonstudios Ende der 1960er Jahre; ${ }^{2}$ über die relative Avanciertheit der Stones und/oder des Produzenten (Jimmy Miller), diese Technologie überhaupt zu verwenden (relativ im Vergleich zu Aufnahmen, die diese technologische Möglichkeit nicht nutzen); über die relative Schlichtheit der Verwendung dieser Technologie, einen bloßen Liegeklang zu erzeugen (relativ im Vergleich dazu, wie viel mehr Möglichkeiten die Studiotechnologie bot, die in anderen Aufnahmen

2 Bereits die zeitliche Zuordnung stellt eine Kontextualisierung dar. 
jener Zeit ausgeschöpft werden); über die Dialektik von Schlichtheit der Form und Komplexität der Mittel (die unabhängig davon besteht, ob die Stones und/oder der Produzent dies intendierten oder nicht), so dass sich die Musik als dialektische gewissermaßen selbst, d.h. in und mit ihren eigenen Klangstrukturen reflektiert; über die darin begründete - und in "Street Fighting Man « sich artikulierende - Dialektik der Moderne, dass Subjekte im Rahmen von technologischem Fortschritt Entscheidungsfreiheit gewinnen und Verfügungsgewalt ausüben.

Geht man hingegen davon aus, dass es sich bei dem besagten Klang um den einer Shehani handelt, würde das Aussagen implizieren über die Verwendung eines fernöstlichen Instruments in westlicher Rockmusik im Zusammenhang mit der seit Mitte der 1960er Jahre festzustellenden Erweiterung ihres Instrumentariums; über den trotz dieser Erweiterung gerade um fernöstliche Instrumente vorhandenen Exotismus; über den soziokulturellen Bedeutungszusammenhang, der der Verwendung fernöstlicher Instrumente eine modische Konnotation des Eskapismus und westlichen Zivilisationsüberdrusses zuschreibt; über eine textlich-musikalische Bedeutungskorrespondenz, die die ambivalente Haltung, wie sie in den Lyrics zum Ausdruck kommt (in den Strophen: Bejahung des Straßenkampfes, im Chorus: dessen Verneinung, weil in »sleepy London« für einen Straßenkämpfer kein Platz sei), zugunsten der Musik einschließlich ihrer eskapistischen Tendenzen entscheidet. $^{3}$

Entgegen dem Eindruck, der aus der vorangegangenen Diskussion entstanden sein könnte, dass das Text/Kontext-Verhältnis so unentwirrbar verflochten ist, dass es ein aussichtsloses Unterfangen wäre, die beiden Aspekte zu separieren, ist das genaue Gegenteil beabsichtigt: Ein Analytiker kommt um die Entscheidung, was sein Gegenstand sei - trotz aller Konsequenzen, die Abgrenzungen mit sich bringen -, nicht herum. Sofern sich Popularmusikforschung als eine Musikwissenschaft versteht (was freilich nicht unabdingbar so sein muss), wäre das Aufgeben klanglicher Strukturen als zentraler Forschungsgegenstand ein Kompetenzverlust; denn geschichtliche Prozesse können auch Historiker untersuchen, gesellschaftliche Netzwerke und Praktiken Soziologen usw. Begreift Popularmusikforschung jedoch Klangstrukturen als Zentrum, ist damit auch der Analysegegenstand erfasst - als Ausgangspunkt, der je nach Fragestellung und Erkenntnisinteresse kontextuell zu erweitern ist. Analyse besteht demzufolge nicht nur in der

3 Um mögliche Horizonte interpretativer Kontextualisierung auszuschreiten, sind beide Ausführungen zu "Street Fighting Man« bewusst überspitzt gezeichnet, was aber gleichzeitig aus ihrer Skizzenhaftigkeit resultiert, die sich durch argumentative Herleitungen einholen ließe. 
Zerlegung größerer Klangeinheiten in kleinere, sondern nimmt bereits eine Zerlegung von Text und Kontext vor. Und so wie musikalische Analyse (im engeren Sinn) größere Klanggebilde in kleinere zerlegt, um deren Funktionieren in gegenseitiger Relation sowie zum Gesamtgebilde synthetisch zu begreifen, separiert Analyse (in einem weiteren Sinn) Text und Kontext, um die ins Auge gefassten Konstitutionsverhältnisse genauer erkennen zu können.

Die Priorisierung der Klangobjekte hätte auch in den sozialen, medialen, historischen etc. Phänomenen, die die populäre Musik ausmachen, ihre Begründung. Dass beispielsweise die Beatlemania oder der Wirbel nach John Lennons Äußerung, die Beatles seien populärer als Jesus, oder die produktive Rezeption der Beatles durch mehrere Britpop-Bands in den 1990er Jahren ohne die Songs der Beatles stattgefunden hätte, darf bezweifelt werden.

»Denn ungeachtet aller soziologischen Rahmenbedingungen hätte die Popmusik niemals ihre Bedeutung gewonnen, wenn ihr nicht ein irreduzibler ästhetischer Erfahrungskern [...] innewohnte, der allen übrigen Phänomenen Zentrum und Ausgangspunkt ist «(Ullmaier 1995: 50).

\section{Zur Methode, oder: All the Tools That're Fit to Splint}

Doch unter welchen Kriterien wäre populäre Musik konkret zu analysieren? Bisherige Äußerungen hierzu haben vor allem die Angemessenheit von Analysemethode und Musik gefordert. Würde dies nicht geleistet, etwa »through the application of inappropriate criteria«, führte dies zu einem »misunderstanding « (Middleton 1990: 103) populärer Musik. Middletons Feststellung beruht im Fall der unpassenden Analyseansätze und Beschreibungsweisen auf der bloßen Übertragung von Kriterien - und damit Werten -, die an einem bestimmten Repertoire gewonnen wurden, auf ein anderes, das diese Kriterien und Werte jedoch nicht teilt. Dem ist zweifellos zuzustimmen. Es wirft aber die Frage auf, wovon sich demgegenüber die passenden Kriterien ableiten ließen. In einem fachhistorischen Rückblick führt Middleton dies an anderer Stelle dahingehend aus:

»Much of the early musicology of pop [...] drew on modes of descriptive and structural analysis, and of rather speculative hermeneutics, that were familiar from the existing traditions of the musicological discipline, as they had been applied to the classical repertoire; the issue here, for many, was whether such 
modes were capable of grasping the pop texts as they were actually understood in the culture or whether, rather, they perhaps misrepresented them « (Middleton 2000: 2).

Als Kriterium von Angemessenheit fungiert damit ein Begriff des tatsächlichen bzw. eigentlichen Verstehens, der durch seine Verortung in einer jeweils zeitgenössischen Kultur als maßgeblich qualifiziert wird. Middleton ist insofern beizupflichten, als die Situierung eines Musikstücks in der Kultur seiner Entstehungs- und ersten Rezeptionszeit unschätzbare Einsichten liefern kann, und zwar sowohl in das Stück als auch in seinen kulturellen Kontext. Doch wenn dies das primäre oder gar ausschließliche Kriterium für angemessenes Analysieren und Verstehen bleiben sollte (was Middletons Aussage nahe legt, auch wenn er in anderen Publikationen das Gegenteil beweist), würde sich eine Forschungsdisziplin einer ganzen Reihe potentieller Fragestellungen entledigen, zu deren Beantwortung Analyse dienen kann. Denn ursprüngliche Entstehungs- und Rezeptionskulturen sind keine Konstanten, die ahistorisch fortdauern, vielmehr unterliegen sie selbst einem historischen Wandel; sie können spätere Verstehensweisen eines Stücks zwar prägen, doch diese wären dann ihrerseits Gegenstand einer Untersuchung, die danach fragen könnte, wie die diskursiven Bedingungen beschaffen sind, dass manche ältere Verstehensweisen in einer späteren Kultur übernommen werden, andere hingegen nicht. Außerdem würde durch das Beibehalten einmal hergeleiteter Kriterien einer zukünftigen Theoriebildung, die sich möglicherweise auf ganz andere als zeitgenössische Kriterien beruft, deswegen aber nicht unbrauchbar sein müssen, der Riegel vorgeschoben werden. (Beispielsweise wurden die Methoden Heinrich Schenkers oder die pitch-class set theory Allan Fortes erst Jahrzehnte nach der Entstehung des Repertoires, auf das sie angewendet werden, [weiter-] entwickelt, stellen aber dennoch, im Rahmen ihrer Möglichkeiten, äußerst brauchbare Analysewerkzeuge dar.)

Daneben ist das Kriterium jenes »eigentlichen Verstehens « auch auf einer wissenschaftstheoretischen Ebene problematisch, weil es ein Objektivitätsideal mit Ausschließlichkeitsanspruch einführt. Darin weist es eine merkwürdige Parallele zu älteren Wissenschaftsparadigmen auf, beispielsweise zur sogenannten objektiven Historik, wie sie sich im Zuge der Bildung der Geschichtswissenschaft im 19. Jahrhundert durchzusetzen begann. Nichts anderes bedeutet der häufig zitierte Ausspruch Leopold von Rankes, einem ihrer Hauptvertreter, dass Geschichtsschreibung lediglich zu zeigen habe, »wie es eigentlich gewesen« (Ranke 1824: VI). Unnötig zu sagen, dass ein solcher Objektivitäts- oder gar Wahrheitsanspruch unter heutigen wissenschaftstheoretischen Prämissen mindestens als fragwürdig gelten muss. 
Doch das Postulat einer angemessenen Kriterienherleitung aus einer damalig zeitgenössischen Kultur birgt ein weiteres Problem. Was würde es bedeuten, eine Musik nur adäquat nach den Modi ihrer zeitgenössischen Kultur beschreiben zu können? Eine Musik nur richtig innerhalb des Kontextes ihrer Produktion, frühen Vermittlung und Rezeption verstehen zu können? Konsequent weitergedacht, wäre dann beispielsweise einem Großteil der Ethnomusikologie oder auch der Mittelalterforschung schlechterdings die Existenzberechtigung abzusprechen. Zwar verbringen Ethnomusikologen Monate, manchmal Jahre in der Kultur, deren Musik sie erforschen möchten, lernen die dort verwendete Sprache, die Bräuche, die sozialen Verhaltensregeln; Mediävisten arbeiten unzählige Traktate und zeitgenössische Berichte durch, aus denen sie Aufschluss gewinnen über das Denken, Fühlen und Handeln zu jener Zeit. Aber sie können sich noch so sehr in eine Kultur hineindenken, -leben und -lesen, am Ende wird immer eine Differenz, eine unüberbrückbare Ferne bleiben, die sie zu einem Fremden macht - und damit für »misrepresentation « anfällig ist. Und wer würde entscheiden, wo Ferne aufhört und Nähe anfängt?

Das Postulat der Angemessenheit zwischen Analysemethode und Gegenstand ist jedoch keineswegs zu negieren, nur wäre zu überlegen, worin die Angemessenheit bestehen soll. Die Nähe einer Methode einschließlich der sie begründenden Kriterien dürfte wohl eher eine sekundäre Rolle spielen. In erster Linie wäre Angemessenheit an den Ergebnissen festzumachen, die eine Methodenanwendung zeitigt. Denn diese mag durchaus fern zum Gegenstand sein: Sofern anschlussfähige, diskursive, neuartige, produktiv weiter zu nutzende Einsichten in den Gegenstand das Resultat sind, ist jede noch so nah- oder abseitige Methode nicht nur angebracht, sondern auch wünschenswert.

\section{Zur grafischen Repräsentation, oder: Bound Sound}

Parallel zu dem eben angesprochenen Kritikpunkt wird auch immer wieder die Verwendung westlicher musikalischer Notation bei der Analyse populärer Musik kritisch in Frage gestellt. Philip Tagg bemerkt hierzu, dass Notation nicht die Hauptquelle für den Analytiker populärer Musik darstellen sollte, denn:

»The reason for this is that while notation may be a viable starting point for much art music analysis, in that it was the only form of storage for over a millennium, popular music [...] is neither conceived nor designed to be 
stored or distributed as notation, a large number of important parameters of musical expression being either difficult or impossible to encode into traditional notation« (Tagg 1982: 41).

Taggs Beobachtung trifft zweifellos zu, und die vielen theoretischen Implikationen, die methodologisch an sie anknüpfen (vgl. z. B. Brackett 2000: 2729; Middleton 1990: 104-106; Wicke 2003: 115-119), unterstreichen ihre Bedeutung. Doch die Unterscheidung, die Taggs Aussage fundiert - in Schrift präskribierte und gespeicherte Kunstmusik einerseits und die Klänge populärer Musik andererseits -, geht in letzter Hinsicht am Kern der Problematik grafischer Repräsentation von musikalischen Klangereignissen vorbei. Setzt man die Praktikabilität von grafischen Mitteln für die musikalische Analyse voraus, ist es gleichgültig, ob es sich um klassische, Pop-, Avantgarde-, frühmittelalterliche, balinesische etc. Musik handelt - sofern in den Klängen etwas analytisch gezeigt werden soll, besteht bei allen die prinzipiell gleiche Aufgabe, sie so oder so grafisch zu repräsentieren. Und in allen Fällen bleiben die grafischen Zeichen - egal ob man eine präskriptive Partitur, eine deskriptive Transkription oder ein computergeneriertes Sonogramm verwendet - angesichts des realisierten und hörbaren Klangs eine nachgerade lächerliche Krücke. Allerdings eine, ohne die man als Analytiker kaum auskommt: Damit man auf musikalische Klänge, die per se prozessual und daher für wissenschaftliche Untersuchungen nur schwer zugänglich sind, zugreifen und aus ihnen Informationen über ihre Eigenschaften gewinnen kann, müssen sie verfügbar gemacht werden, auch zum Zweck ihrer Kommunizierbarkeit.

Eine häufig anzutreffende Behauptung, die die Unterscheidung nichtnotierter populärer Musik und notierter westlicher Kunstmusik stützt, dass deren Notation ihre wesentlichen Eigenschaften abbilde, trifft allenfalls auf einer theoretischen Ebene zu. Jeder, der einmal eine Partitur gehört hat (sic), die über ein MIDI-Programm abgespielt wurde, wird zugeben, dass die Wiedergabe exakter Tonhöhen und Dauernverhältnisse wenig mit der musikalischen Praxis zu tun hat. Und dass die historische Musikwissenschaft und Musiktheorie nach einer »notational centricity« (Tagg) orientiert sind, unter Vernachlässigung prozessualer, performativer Aspekte, ist denn auch zu Recht kritisiert (Middleton 1990: 105f.) und in diesen Disziplinen erst in jüngerer Zeit ernsthaft thematisiert worden (vgl. z. B. Abbate 2004). Mit anderen Worten: Die westliche Kunstmusik beruht wie andere Musikarten auch auf mündlichen Überlieferungen, die sich zwar graduell, nicht aber kategorial unterscheiden, so dass das Problem der Notation nicht in einer Ver- 
schiedenartigkeit von Klängen unterschiedlicher Musikpraktiken, ${ }^{4}$ sondern in einer unhintergehbaren Eigenschaft grafischer Repräsentation begründet liegt: ihrer operativen Reduktivität.

Jede Transformation von phänomenalen Gegebenheiten in eine grafische Form zum Zweck ihrer Symbolisierung beinhaltet reduktive Schritte, weil das Phänomen eine so große Informationsmenge aufweist, dass sie gesamthaft nicht in die Grafik übertragbar ist oder nicht übertragen werden soll, da sonst der Zweck der Grafik getrübt würde. Die grafische Symbolisierung soll nur jene Informationen enthalten, die für den Zweck ihrer Verwendung hinreichend sind: »No system of transcription, mechanical or otherwise, can preserve all of a musical example accurately and it is up to the transcriber to select or emphasize pertinent parts of the entire configuration« (Garfias 1964: 233). Es bedarf allerdings der vorausgesetzten Übereinkunft zwischen den Herstellern und Nutzern grafischer Repräsentationen, dass es sich um eine zweckhafte Reduktivität handelt. Würde beispielsweise ein Nutzer dem Hersteller einer Straßenkarte vorwerfen, er habe nicht jede minimale Straßenbiegung abgebildet, müsste sich der Nutzer seinerseits vorwerfen lassen, dass er den Zweck einer solchen Straßenkarte nicht verstanden habe. Denn die Abbildung jeder Biegung würde einerseits die Karte unleserlich machen und andererseits überflüssige bzw. irrelevante Informationen enthalten, beides bezogen auf den Zweck der Orientierung in relativ großen räumlichen Verhältnissen bei relativ hohen Geschwindigkeiten des automobilen Verkehrs.

Dass grafische Repräsentationen von Klängen - egal ob prä- oder deskriptiv - grundsätzlich reduktiv sind, wäre insofern nicht weiter der Rede wert, weil selbstverständlich. Allerdings ist zu betonen, dass die Herstellung grafischer Repräsentationen oder das selektive Heranziehen bestehender Notationen selbst schon als analytischer Schritt zu betrachten ist. Dementsprechend wäre zu folgern, dass Transkriptionen und andere Grafiken nicht primär aus einer jeweiligen Musikart abgeleitet werden können, sondern in erster Linie der analytischen Fragestellung und der zum Zweck ihrer Beantwortung passenden Mittel folgen sollten. Und diese Orientierung an der Fra-

4 Es gehört zu den Merkwürdigkeiten (die die Vermutung stützt, dass einige Popularmusikforscher sich hier von einer am Problem vorbeigehenden Intention der Abgrenzung gegenüber anderen musikwissenschaftlichen Disziplinen leiten lassen), dass zwar die Verwendung westlicher musikalischer Notation für populäre Musik problematisiert wurde, nicht aber die Verwendung der Buchstabenschrift für das gleichfalls nur klanglich vorliegende Phänomen gesungener Texte; gedruckte Lyrics sind aber, wie Sheets, nachträgliche Transkriptionen. Mir ist nur eine Untersuchung bekannt (Senn 2007), die diese Thematik, mittels der Werkzeuge der International Phonetic Association, überhaupt und überzeugend angeht. 
gestellung bestimmt auch, welche Eigenschaften eines Komplexes grafisch auf welche Weise dargestellt werden.

\section{Zum Anspruch, oder: »For What It's Worth«}

Die Reduktion ist kein Problem der grafischen Repräsentation allein, sondern eine grundsätzliche Implikation musikalischer Analyse. Peter Wicke hat, in einer methodologischen Kritik mehrerer Publikationen von Walter Everett, daher zu Recht auf ihren nur partikular geltenden Anspruch verwiesen:

»Die Reduktion einer ebenso komplexen wie widersprüchlichen Musikpraxis auf solche Erscheinungen, die sich in Begriffen der klassisch-romantischen Musiktheorie schenkerscher Prägung fassen lassen, ist jedoch ein methodologisch höchst fragwürdiges Unternehmen« (Wicke 2003: 113). ${ }^{5}$

Das Unbehagen, das Wicke angesichts der Anwendung von Methoden artikuliert, die auf den Theorien Heinrich Schenkers beruhen, wäre prinzipiell an jeder Methodenanwendung zu kritisieren: als Reduktion einer je mehr oder weniger komplexen Musikpraxis. Die auf Schenkers Theorie fußenden Ansätze erfassen Tonhöhenaspekte und Stimmführungen in einem größeren formalen Zusammenhang; Harmonieanalyse erfasst nur die zusammenklingenden Tonhöhenbeziehung und die Relationen dieser Zusammenklänge untereinander und zu einem Tonzentrum; Rhythmusanalyse ist nur auf die kleindimensionalen zeitlichen Relationen von Klängen, Formanalyse nur auf die größer dimensionierten zeitlichen Relationen von Klangkomplexen ausgerichtet; Klanganalyse mittels Sonogrammen erfasst zunächst nur die akustischen Eigenschaften von Klängen im zeitlichen Verlauf; usw.

Solange sich ein Analytiker der begrenzten Reichweite seiner Ansätze bewusst ist und dementsprechend zum einen die Ergebnisse seiner Analyse nicht verabsolutiert oder zum anderen mehrere Analyseansätze anwendet, um seinen analytischen Gesichtskreis auszuweiten, stellt die reduktive Geltung von Analyse auch kein Problem dar. Dies wäre ferner dahingehend zu betonen, dass Analyse ohnehin von einer Fragestellung geleitet wird, zu deren Beantwortung sie lediglich dient. (Nichts ist unanalytischer, als der isolierte Vorsatz, »mal ein Stück zu analysieren«.) Das jeweilige Erkenntnisinteresse am Gegenstand, das diesem gegenüber immer nur partikular sein kann, bedingt die Analysemethode, nicht umgekehrt.

5 Der grundsätzliche Gedanke Wickes zum beschränkten Anspruch von Analyse ist zu unterstreichen, seine Kritik an Everett teile ich jedoch nicht. 
Daran sowie an die eingangs gemachte Beobachtung anknüpfend, dass Musikstücke keine unveränderlichen Entitäten darstellen, ist schließlich auf die dynamische Prozessualität des Analysierens zu verweisen. Denn um zu erkennen, welche Methode zu einem Gegenstand passen könnte, muss ein Analytiker den Gegenstand bereits in irgendeiner Weise als solchen erkannt haben, d.h. er muss wenigstens rudimentäre analytische Handlungen durchgeführt haben. Im einfachsten Fall heißt das, ihn wahrgenommen zu haben. Und Wahrnehmung ist nicht nur ein physiologisch-sensorischer Vorgang, auch nicht ein bloß perzipierendes Hören, sondern beinhaltet, wie rudimentär auch immer, analytische, also unterscheidende, zuordnende, bewertende Handlungsanteile. Angenommen, nach einer Wahrnehmung setzte sich ein Analytiker daran, einen musikalischen Gegenstand (weiter) zu analysieren, dann hört dieser Austausch zwischen ihm und dem Gegenstand ja nicht auf. Denn wie oft passiert es beim Analysieren, dass man in einem späteren Stadium auf Dinge aufmerksam wird, die man zunächst gar nicht wahrgenommen hatte; folglich geht die Analyse weiter in diese Richtung, wofür man aber eventuell einen anderen Ansatz oder gar eine andere Methode anwendet, und wieder gelangt man zu weiteren Aspekten - vielleicht durch Hinzuziehung von Quellen -, die den Gegenstand in einem anderen Licht darstellen, und um die Gründe dieser Belichtung herauszufinden, verfolgt man wiederum einen anderen Ansatz. So verstanden, ist Analyse weder eine Einbahnstraße des Verstehens - vom Gegenstand durch die Methode zum Ergebnis - noch eine einmalige Handlung, sondern vollzieht sich in fortwährender Interaktion zwischen Gegenstand und Analytiker als dialogischer Prozess, der prinzipiell unabschließbar ist. Hans-Georg Gadamer begründete diesen Prozess (in Anlehnung an Robin George Collingwood) in einer »Logik von Frage und Antwort« (Gadamer 1975: 351ff.), der alles hermeneutische Tun folge. Für den Fortgang des Prozesses ist es entscheidend, dass ein jeweils erreichtes Verständnis nur als »Vorgriff « oder »Vorurteil der Vollkommenheit « (ebd.: 277f.) gelten kann. Hat demnach Gadamers »hermeneutischer Zirkel«, zumindest begrifflich, einen holistischen Fluchtpunkt, so ist gegenüber dieser hierarchischen eine alternierende Verstehensbewegung anzunehmen, weil sie über die Partikularität von Analyse nicht hinausgreifen kann: Analyse als dialogischer Prozess resultiert nicht in einem transzendentalen Ziel, sondern ist durch der Jeweiligkeit ihrer (methodischen) Perspektiven bedingt, die ein Analytiker wechselseitig einnimmt. Ganzheit gibt es analytisch nicht, und keine Musik ist zu Ende analysierbar. 


\title{
Literatur
}

Abbate, Carolyn (2004). »Music: Drastic or Gnostic?« In: Critical Inquiry 30, S. 505536.

Brackett, David (2000). Interpreting Popular Music. Berkeley: University of California Press [zuerst 1995].

Gadamer, Hans-Georg (1975). Wahrheit und Methode. Grundzüge einer philosophischen Hermeneutik. Tübingen: Mohr (4. Aufl.).

Garfias, Robert (1964). »Transcription I.«In: Ethnomusicology 8, S. 233-240.

Goer, Lydia (1992). The Imaginary Museum of Musical Works: An Essay in the Philosophy of Music. Oxford: Clarendon Press.

Hartwich-Wiechell, Dörte (1974). Pop-Musik. Analysen und Interpretationen. Köln: Volk.

Middleton, Richard (1990). Studying Popular Music. Milton Keynes, Philadelphia, PA: Open University Press.

Middleton, Richard (2000). "Introduction: Locating the Popular Music Text.« In: Reading Pop: Approaches to Textual Analysis in Popular Music. Hg. v. Richard Middleton. Oxford: Oxford University Press, S. 1-19.

Ranke, Leopold (1824). Geschichten der romanischen und germanischen Völker von 1494 bis 1535, Bd. 1. Berlin, Leipzig: G. Reimer.

Seel, Martin (2003). Ästhetik des Erscheinens. Frankfurt/M.: Suhrkamp.

Senn, Olivier (2007). Die Analyse von Tonaufnahmen. Konzepte und Methoden zur musikwissenschaftlichen Analyse von Tonaufnahmen - dargestellt an Sarah Vaughans Einspielung des Musicalhits >My Favorite Things von 1961. Zürich: Universität Zürich [greifbar über die Dissertationsdatenbank http://www. dissertationen.unizh.ch].

Tagg, Philip (1982). »Analysing Popular Music: Theory, Method and Practice.« In: Popular Music 2, S. 37-67.

Ullmaier, Johannes (1995). Pop Shoot Pop. Über Historisierung und Kanonbildung in der Popmusik. Rüsselsheim: Frank Hofmann.

Wicke, Peter (2003). »Popmusik in der Analyse.«In: Acta Musicologica 75, S. 107126.

\begin{abstract}
In methodological debates on the analysis of popular music some topics have gained broad attention. Four of these topics are discussed in this essay: the matter of the analytical object; the demand of an adequacy between method and analyzed music; the problem of the graphical representation of sounds; and eventually the claim what analysis can or should achieve. In the aforementioned debates, great efforts have been made to sharpen the profile of popular music studies by distinguishing it from other musicological disciplines. While discussing the methodological topics this essay also takes into account the gains and losses of that distinction.
\end{abstract}


Bereitgestellt von | Universitaetsbibliothek Basel

Angemeldet

Heruntergeladen am | 21.09.18 17:28 